\title{
Orai3 is a predictive marker of metastasis and survival in resectable lung adenocarcinoma
}

\author{
Nazim Benzerdjeb ${ }^{1,2}$, Henri Sevestre ${ }^{2}$, Ahmed Ahidouch ${ }^{2,3}$, Halima Ouadid-Ahidouch ${ }^{1}$ \\ ${ }^{1}$ Laboratory of Cellular and Molecular Physiology, LPCM: EA 4667, SFR CAP-SANTE (FED 4231), UFR of Sciences, Amiens, \\ France \\ ${ }^{2}$ Department of Pathology, Amiens University Hospital, Amiens, France \\ ${ }^{3}$ Department of Biology, Ibn Zohr University, Agadir, Morocco \\ Correspondence to: Halima Ouadid-Ahidouch, email: halima.ahidouch-ouadid@u-picardie.fr \\ Nazim Benzerdjeb, email: nazim.benz@gmail.com \\ Keywords: orai3, lung adenocarcinoma, prognostic marker, survival
}

Received: June 11,2016 Accepted: October 17, $2016 \quad$ Published: November 07, 2016

\section{ABSTRACT}

Orai3 channel has emerged as important player in malignant transformation. Indeed, its expression is increased in cancer and favors cell proliferation and survival by permitting calcium influx. In this study, Orai 3 was overexpressed in lung adenocarcinoma as compared to their matched non-tumour samples and was associated with tumoural aggressiveness. Moreover, its expression was associated with estrogen receptor alpha (ERa) expression and visceral pleural invasion in multivariate analysis. Furthermore, both the overall survival (OS) median and the metastasis free survival (MFS) median of tumors with high Orai3 expression were lower than in low Orai3 expression regardless of cancer stage ( 35.01 months vs. 51.11 months for OS and 46.01 months vs. 62.04 months for MFS). In conclusion, Orai 3 protein level constitutes an independent prognostic marker in lung adenocarcinoma, and a novel prognostic marker that could help selecting the patients with worst prognosis to be treated with adjuvant chemotherapy in resectable stage.

\section{INTRODUCTION}

Lung cancer, particularly adenocarcinoma, is the leading cause of cancer deaths worldwide [1] and the most common histologic type of lung cancer [2]. Moreover, lung adenocarcinoma is known to be a very heterogeneous tumour, with over $90 \%$ of resected tumours [3]. For this reason, the accuracy of patient prognosis prediction of the international staging system is still insufficient with recurrence rate after radical surgery around $40 \%$ of early stage patients [4]. The use of chemotherapy [CT] after radical surgery is justified by increasing overall survival from 60 to $64 \%$ [5-7]. As the benefit of chemotherapy is moderate, it is important to select appropriate patients with resectable tumours. The chemotherapy is recommended for patients with stage II or III of lung adenocarcinoma, and also for patients with stage Ib with high risk of recurrence. Thus, it is necessary to implement new prognostic markers of high risk for recurrence that could help to identify the patients with worst prognosis [4]. Some histologic and immunohistochemical biomarkers have been proposed. In 2011, a lung adenocarcinoma classification based on predominant histologic pattern (lepidic, papillary, acinar, solid and micropapillary) has been proposed and is currently accepted [8]. The predominant histologic pattern: low (lepidic), intermediate (papillary, acinar) and high risk (solid, micropapillary), constitutes a predictive factor for recurrence in resectable stage lung adenocarcinoma [4]. Others immunohistochemical biomarkers, such as estrogen receptor alpha expression (ER $\alpha$ ) expression, are associated with a poor prognosis in lung adenocarcinoma $[9,10]$. Moreover, ER $\alpha$ also is an independent factor of recurrence in pT1a lung adenocarcinomas [11]. But few studies have discussed a biomarker involved in calcium metabolism.

Calcium $\left(\mathrm{Ca}^{2+}\right)$ plays a crucial role in regulating several processes such as cell proliferation $[12,13]$ and apoptosis [14]. The scientific community, including our group, has shown an interest in Orai channels family because these latter are known to play a major pathway of 
calcium influx in epithelial cells. Three Orai isoforms have been characterized: Orai1, Orai2 and Orai3. Orai3 is a unique channel whose expression is restricted to mammals [15]. Several studies have reported the involvement of Orai3 in the complex machinery of carcinogenesis including breast, prostate and lung cancer [16-18]. Moreover, the expression of Orai3 is regulated by several factors. Indeed, in breast cancer, Orai3 is regulated by the $\mathrm{ER} \alpha$, thereby conferring apoptosis resistance and cell proliferation $[16,19,20]$. Indeed, silencing of ER $\alpha$ caused a significant decrease of Orai3 expression, calcium influx, and cell proliferation in vitro [19]. Furthermore, epidermal growth factor (EGF) stimulates $\mathrm{Ca}^{2+}$ influx into estrogen receptor-positive MCF-7 cells through Orai3 [16]. In lung cancer, we have previously reported an overexpression of Orai3 in a small cohort of adenocarcinoma $(\mathrm{N}=60)$, and its role in cell proliferation and $\mathrm{Ca}^{2+}$ influx in lung cancer cell lines [21]. Here we evaluated the immunohistochemical expression of Orai3 in a large cohort of lung adenocarcinoma samples $(\mathrm{N}=200)$ taking into account their clinic-pathologic features (tobacco exposure, tumour necrosis, visceral pleural invasion, 2011 classification of lung adenocarcinoma and stage), TTF1 expression, tumoural aggressiveness (ER $\alpha$ expression, KRAS and EGFR mutations) and prognostic significance was further evaluated.

\section{RESULTS}

\section{Orai3 is overexpressed in lung cancer and associated with tumoural aggressiveness}

First, we confirmed that Orai3 is overexpressed in lung adenocarcinomas (Figure 1A). Among the 200 cases of tumour tissues matched with non-tumour tissues tested, the score of Orai3 staining in tumour tissues was higher than in matched non-tumour ones (non-tumour tissues: 0.3 \pm 0.04 vs. tumour tissues: $0.66 \pm 0.005, p<0.05$; Wilcoxon signed-rank test). We found also that the mRNA for Orai3 was strongly overexpressed in tumour tissues (4.08 \pm 0.54 folds higher than in non-tumour tissues, Figure 1B, $p<0.05$; Mann-Whitney). The expression of Orail and Orai2 was also analyzed by immunohistochemistry in the same tissues samples $(\mathrm{N}=200)$. In contrast to the over-expression of Orai3 in $66.5 \%(\mathrm{~N}=133 / 200$, $\mathrm{H}$-score mean $=0.66)$, Orai1 and Orai2 overexpression were found in $31 \%(\mathrm{~N}=62 / 200, \mathrm{H}$-score mean $=0.20)$ and $32 \%(\mathrm{~N}=64 / 200, \mathrm{H}$-score mean $=0.19)$ cases of the tissue samples studied respectively. Representative expressions of Orai1 and Orai2 in cancerous human lung tissues are showed in the Supplementary Figure S1.

We further investigate whether the expression of Orai3 is associated to tumour aggressiveness. To do this, we compared the score staining of Orai3 in invasive lepidic, papillary, acinar and solid adenocarcinomas. The staining of Orai3 was low in non-tumour lung, and in invasive predominant lepidic and papillary adenocarcinoma (Figure 1C-1D), was moderate in infiltrating acinar adenocarcinoma (Figure 1E), and high in infiltrating solid adenocarcinoma (Figure 1F). We quantified the score staining of Orai3 in two groups: group 1 (invasive adenocarcinoma lepidic, papillary and acinar predominant) and group 2 (invasive adenocarcinoma solid and micropapillary predominant). Score staining of Orai3 was higher in group 2 than in group 1 (Figure $1 \mathrm{G}$ : 0.64 \pm 0.007 for the group $1, \mathrm{~N}=133 / 180$ vs. $0.95 \pm 0.02$ for the group 2, $\mathrm{N}=47 / 180 ; p<0.001$ ). Thus, a high expression of Orai3 was significantly associated with solid and micropapillary predominant invasive adenocarcinomas which are the more aggressive of lung adenocarcinoma. These data suggest that Orai3 expression is associated with tumour aggressiveness.

\section{Clinical significance of Orai3 expression in lung cancer patients}

The relationship of Orai3 expression and clinicopathological factors from 200 lung cancer patients is presented in Table 1. Univariate and multivariate regression analysis revealed that high expression of Orai3 was significantly associated with tumour necrosis, tobacco use, TTF1 expression, and visceral pleural invasion (OR: 5.03) and ER $\alpha$ expression (OR: 5.29) respectively (Table 1). Moreover, of the 59 samples exhibiting high expression of Orai3, a high ER $\alpha$ was found in all samples $(100 \%)$. A representative immunohistochemistry staining is shown in Supplementary Figure S2.

However, no association between score staining of Orai3 and EGFR mutation (9/129), KRAS mutation $(18 / 52)$ and stage TNM was found (Table 1). The overall survival (OS) and metastasis free survival (MFS) rate in the high Orai3 expression group was significantly lower in comparison with the low Orai3 expression group (Figure 2A). For OS, the median follow-up was 35.01 months vs. 51.11 months for the high and low Orai3 expressions respectively (Figure $2 \mathrm{~A}, p<0.05$; log rank test). For the metastasis free survival, the median was 46.01 months vs. 62.04 months for the high and low Orai3 expressions respectively (Figure $2 \mathrm{~B}, p<0.05, \log$ rank test). Multivariate regression analysis revealed that high expression of Orai3 is an independent prognostic factor for lung cancer outcome, as the tumour stage (Table 2).

\section{Expression of Orai3 and survival in stages Ib and II-III}

The chemotherapy is recommended for patients with stage II or III of lung adenocarcinoma, and also for patients with stage $\mathrm{Ib}$ with high risk of recurrence. As Orai3 confers survival of cancer cells [21], we evaluated the expression of Orai3 in stage Ib and II-III. 

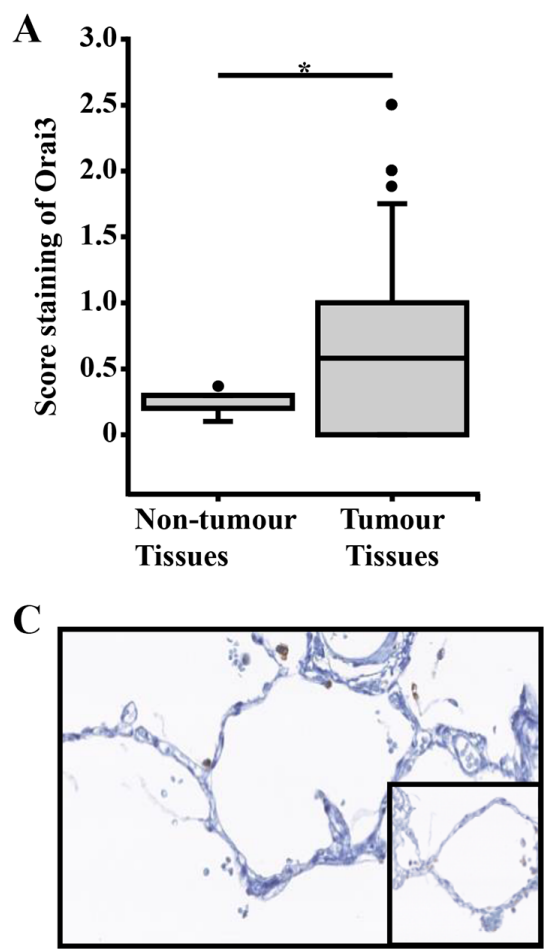

$\mathbf{E}$

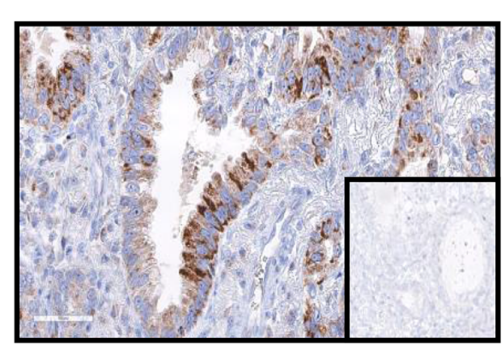

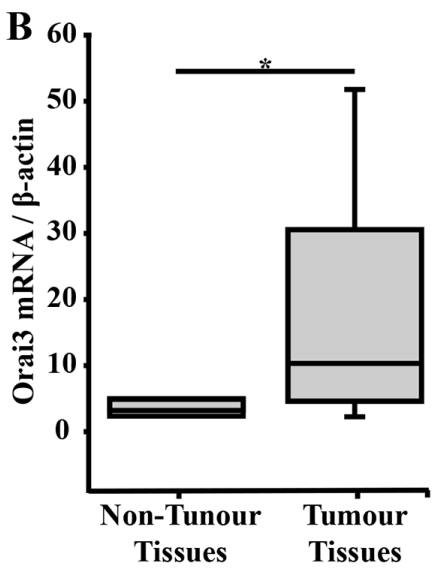

D

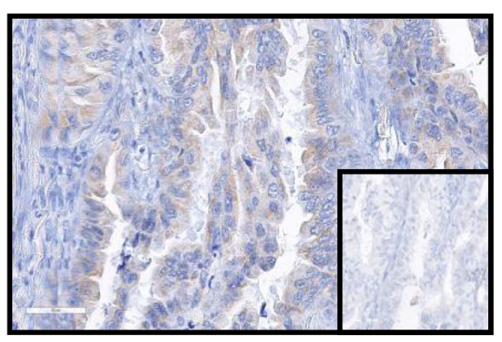

F

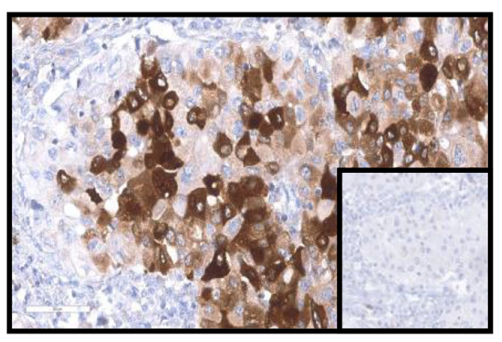

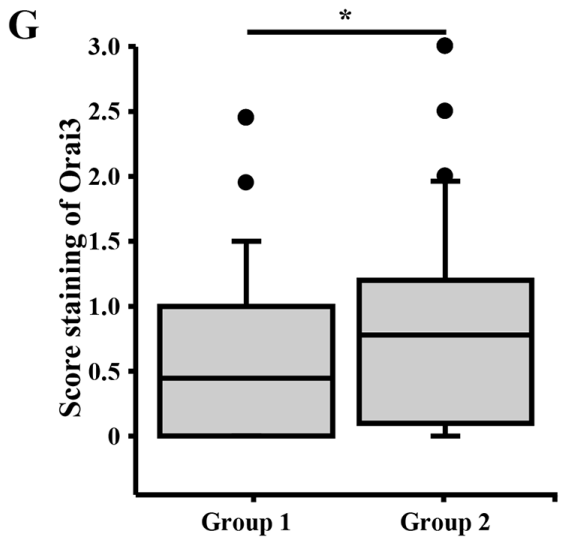

Figure 1: Immunohistochemical stainings of Orai3 in lung adenocarcinoma tissues according to the lung adenocarcinoma classification and relative quantity of Orai3 mRNA in lung adenocarcinoma tissues. A. In 200 specimens, the score of Orai3 was higher in tumour tissues than in matched non-tumour tissues $(p<0.05$, Wilcoxon signed-rank test). B. Orai3 mRNA expression in 10 formalin-fixed, paraffin-embedded lung adenocarcinoma and non-tumour tissue samples $(p<0.05$, Mann-Whitney test). C. Normal lung tissue showed a few staining of Orai3 in all the epithelium cells (x200). D. Lung adenocarcinoma showed a few staining of Orai3 in invasive adenocarcinoma lepidic and papillary predominant (x200). E. Lung adenocarcinoma showed a moderate Orai3 staining in infiltrating adenocarcinoma acinar predominant (x200). F. Lung adenocarcinoma showed high Orai3 staining in infiltrating adenocarcinoma solid predominant (x200); inserts show negative controls obtained by omitting the primary antibody. G. The assessment of Orai3 score was performed on 180 invasive adenocarcinoma samples. The group 1 includes samples of invasive adenocarcinoma lepidic, papillary and acinar predominant. The group 2 includes samples of invasive adenocarcinoma solid and micropapillary predominant. 
Table 1: Association between Orai3 expression and clinico-pathological characteristics of the lung adenocarcinoma $(\mathrm{N}=\mathbf{2 0 0})$

\begin{tabular}{|c|c|c|c|c|}
\hline \multirow[t]{2}{*}{ Variables } & \multicolumn{2}{|c|}{ Univariate analysis } & \multicolumn{2}{|c|}{ Multivariate analysis } \\
\hline & $\mathrm{OR}+95 \% \mathrm{CI}$ & $p$ value* & $\mathrm{OR}+95 \% \mathrm{CI}$ & $p$ value* \\
\hline Necrosis (p vs. a) & $2.18[1.12-4.28]$ & 0.023 & $1.15[0.60-2.20]$ & 0.66 \\
\hline Tobacco use (s vs. nos) & $1.67[0.99-2.81]$ & 0.04 & $1.89[0.473-8.03]$ & 0.39 \\
\hline VPI (p vs. a) & $5.16[2.30-11.55]$ & $<0.001$ & $4.68[1.9-11.51]$ & $<0.001$ \\
\hline Histologic classification $\dagger(\mathrm{M}-\mathrm{S}$ vs. L-P-A) & $3.15[1.42-6.98]$ & 0.005 & $1.40[0.47-4.11]$ & 0.55 \\
\hline TTF1 $(>1 \%$ vs. $<1 \%)$ & $2.08[1.01-4.26]$ & 0.04 & $2.33[0.91-5.97]$ & 0.08 \\
\hline $\operatorname{ER} \alpha(>1 \%$ vs. $<1 \%)$ & $7.72[3.42-17.39]$ & $<0.001$ & $5.29[2.05-13.67]$ & $<0.001$ \\
\hline EGFR (MT vs. WT) & $0.72[0.178-2.99]$ & 0.66 & & \\
\hline KRAS (MT vs. WT) & $3.03[0.36-25.21]$ & 0.30 & & \\
\hline Stage (IIIa-IV vs. I-II) & $1.89[0.89-4.01]$ & 0.097 & & \\
\hline
\end{tabular}

* Univariate and multivariate logistic regression model

$\uparrow$ Novel classification of lung adenocarcinoma from WHO 2015

Abbreviation: a: absence; ER $\alpha$ : estrogen receptor alpha; MT: mutant type; L-P-S-M-S: invasive adenocarcinoma predominant lepidic, papillary, acinar, micropapillary and solid; nos: non-smokers; OR: Odd Ratio; p: presence; s: smokers; TTF1: thyroid transcription factor 1; VPI: visceral pleural invasion; WT: wild type.

For Ib stage, there was no significant impact of Orai3 expression on OS (Figure 2C). For MFS, the median of high Orai3 expression group was lower than low Orai3 expression group (Figure 2D: 36.64 months vs. $>80$ months, $p<0.05$; log rank test). Univariate Cox proportional hazards analysis also showed a negative impact of Orai3 expression on MFS for patients with stage Ib (Table 3: HR: 8.01, $p=0.049$ ), while no significant difference for OS was found (Table 3). For II-IIIa tumour stages, a negative impact of Orai3 expression was observed on OS (Figure 2E, median 25.60 months for high Orai3 vs. 50.73 months for low Orai3 expression, $p=0.02$; log rank test), but not on MFS (Figure 2F). The multivariate Cox proportional hazards model confirms the negative impact of high Orai3 expression for the overall survival of patients with stages II-IIIa (Table 4: HR: 7.16, $p=0.008)$. No significant difference was observed for MFS.

\section{DISCUSSION}

Here, we confirm the association of the Orai3 expression with invasive adenocarcinoma, micropapillary and solid predominant subtypes, in a large cohort of patients of lung cancer. Moreover, we report, for the first time, that high expression of Orai3 is an independent prognostic factor for lung adenocarcinomas. The expression of Orai3 has been evaluated using immunohistochemistry, which is the more relevant technique for heterogeneous tumour [22]. Indeed, in previous studies lung adenocarcinoma exhibited higher heterogeneity [23], and intra-tumor variation of expression in lung cancer was reported by immunohistochemistry [24]. The excellent specificity of Orai3 antibody used in this work has been previously reported [21]. Moreover, the overexpression of Orai3 in lung tissue was also confirmed by qPCR on the same samples embedded by paraffin.

The role of Orai3 in cell proliferation and calcium homeostasis in lung cancer cell lines was previously reported by our group [21]. Indeed, silencing of Orai3 (using siRNA against Orai3) accumulates cells in G0/ G1 phase of the cell cycle, reduces calcium entry leading to cell cycle arrest and cell proliferation inhibition [21]. Studies on the association between the expression of ion channels and the prognosis of lung adenocarcinoma patients emerge. Recently, a transcriptomic analysis has compared the expression of ion channel encoding genes of normal and lung adenocarcinoma tissues. 37 ion channels genes were identified as being differentially expressed between the two groups [25]. However, the Orai isoforms were not assessed in this study. The authors have established a risk score based on the expression of the differentially expressed genes in order to investigate the prognostic of such ion channels genes. Using multivariate analysis, they found that the risk score is an independent prognostic factor for survival [25]. Moreover, they found the risk score was higher for ever-smokers than for neversmokers [25]. The same group has reported that VDAC1 (voltage-dependent anion channel type 1) is associated with shorter overall survival and turned out to be an independent prognostic factor of recurrence in multivariate analysis [26]. Our results show that high Orai3 expression 
Overall survival

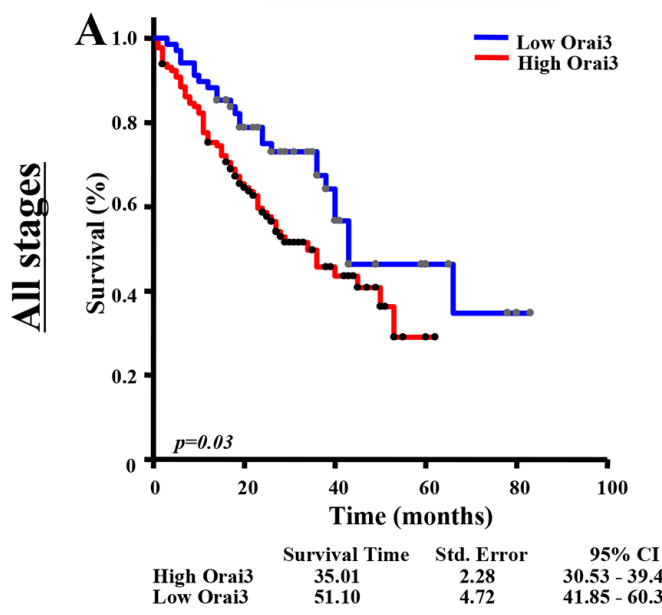

\section{Metastasis free survival}
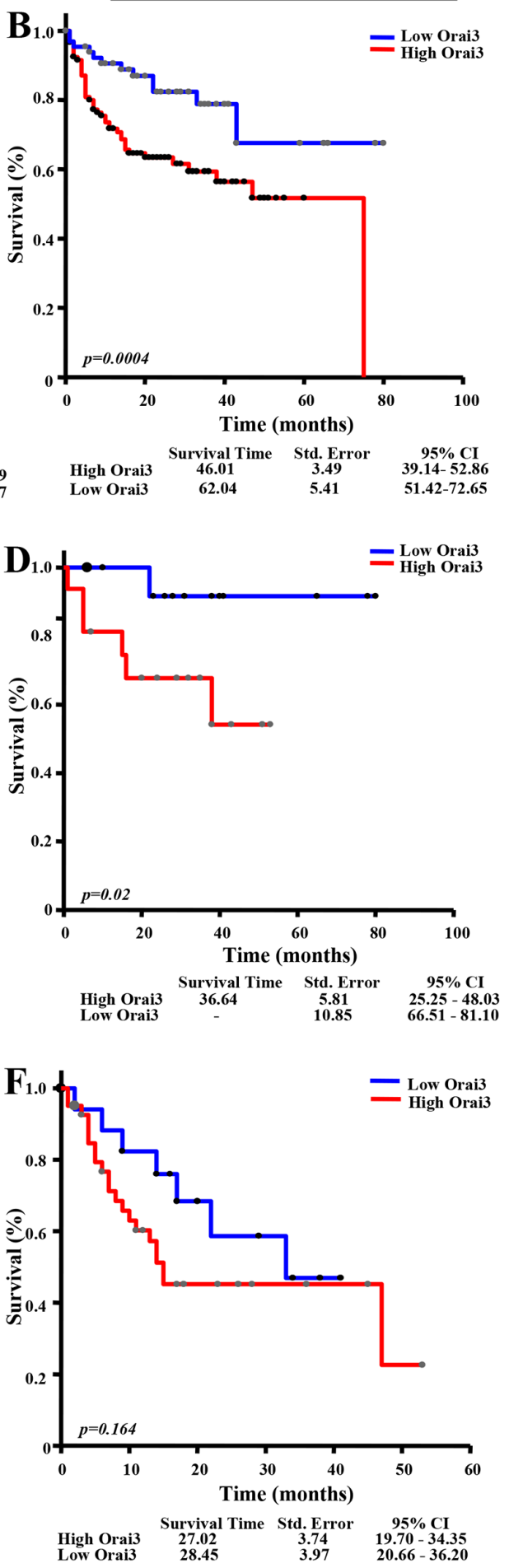

Figure 2: Association of Orai3 expression with survival. (A-B) All stages ( $N=200)$ : A. Overall survival of patients with high Orai3 expression compared with low Orai3 expression $(p<0.05$, Log rank test). B. Metastasis free survival of patients with high Orai3 expression compared with low Orai3 expression $(p<0.05$, Log rank test). (C-D) With stage Ib disease $(\mathrm{N}=32)$ : C. Overall survival of patients with high Orai3 expression compared with low Orai3 expression (ns; Log rank test). D. Metastasis free survival of patients with high Orai3 expression compared with low Orai3 expression ( $p<0.05$, Log rank test). (E-F) With stage II-IIIa disease ( $N=62)$ : E. Overall survival of patients with high Orai3 expression compared with low Orai3 expression $(p<0.05$, Log rank test). F. Metastasis free survival of patients with high Orai3 expression compared with low Orai3 expression (ns, Log rank test). Low Orai3 expression group is shown as blue solid line; high Orai3 expression group is shown as red solid line. Abbreviations: 95\% CI: 95\% confidence interval; ns: non-significant; Std. Error: standard error. 
Table 2: Cox regression analysis for OS and MFS for adenocarcinoma patients for the whole cohort $(\mathrm{N}=200)$

\begin{tabular}{|c|c|c|c|c|c|c|c|c|}
\hline \multirow[t]{3}{*}{ Variable } & \multicolumn{4}{|c|}{ OS } & \multicolumn{4}{|c|}{ MPF } \\
\hline & \multicolumn{2}{|c|}{ Univariate analysis } & \multicolumn{2}{|c|}{ Multivariate Analysis } & \multicolumn{2}{|c|}{ Univariate analysis } & \multicolumn{2}{|c|}{ Multivariate analysis } \\
\hline & HR $[95 \% \mathrm{CI}]$ & $p$ value & HR $[95 \% C I]$ & $p$ value & HR $[95 \% C I]$ & $p$ value & HR [95\%CI] & $p$ value \\
\hline Orai3 (high vs. low) & $1.66[1.04-2.63]$ & 0.033 & $1.55[1.02-2.23]$ & 0.045 & $2.82[1.54-5.17]$ & 0.001 & $2.73[1.45-5.16]$ & 0.002 \\
\hline Gender $(\mathrm{M} v s . \mathrm{F})$ & $0.85[0.45-1.60]$ & 0.570 & & & $0.82[0.44-1.54]$ & 0.516 & & \\
\hline Stage (I-IV vs. Ia) & & $<0.001$ & & $<0.001$ & & $<0.001$ & & $<0.001$ \\
\hline $\mathrm{Ib} v s . \mathrm{Ia}$ & $0.67[0.33-1.37]$ & & $0.82[0.33-2.04]$ & & $1.29[0.57-2.92]$ & & $1.94[0.85-4.45]$ & \\
\hline IIa vs. Ia & $0.87[0.36-2.07]$ & & $0.86[0.29-2.54]$ & & $2.85[1.23-6.64]$ & & $3.25[1.36-7.77]$ & \\
\hline IIb vs. Ia & $1.73[0.80-3.76]$ & & $1.79[0.66-4.80]$ & & $3.08[1.28-7.45]$ & & $3.57[1.44-8.81]$ & \\
\hline IIIa $v s$. Ia & $2.84[1.62-4.96]$ & & $3.01[1.58-5.73]$ & & $3.89[1.95-7.77]$ & & $2.51[1.18-5.34]$ & \\
\hline IV vs. Ia & $4.03[2.09-7.79]$ & & $4.73[2.32-9.63]$ & & $10.94[5.23-2.90]$ & & $10.86[5.01-3.50]$ & \\
\hline Necrosis (p vs. a) & $1.48[0.91-2.42]$ & 0.116 & $1.06[0.78-1.44]$ & 0.609 & $1.72[1.06-2.80]$ & 0.029 & $1.53[0.92-2.54]$ & 0.099 \\
\hline Age & $0.99[0.97-1.02]$ & 0.884 & & & $0.99[0.97-1.02]$ & 0.369 & & \\
\hline Tobacco use (s vs. nos) & $1.86[0.58-5.96]$ & 0.740 & & & $0.94[0.36-2.47]$ & 0.590 & & \\
\hline
\end{tabular}

Abbreviations: a: absence; 95\%CI: 95\% confidence interval; F: female; HR: Hazard Ratio; M: male; MFS: metastasis free survival; ns: non-significant; nos: non-smokers; OS: overall survival; p: presence; s: smokers.

Table 3: Cox regression analysis for OS and MFS for adenocarcinoma patients for patients with Ib stage (N=32)

\begin{tabular}{|c|c|c|c|c|c|c|c|c|}
\hline \multirow[t]{3}{*}{ Variable } & \multicolumn{4}{|c|}{ OS } & \multicolumn{4}{|c|}{ MPF } \\
\hline & \multicolumn{2}{|c|}{ Univariate Analysis } & \multicolumn{2}{|c|}{ Multivariate analysis } & \multicolumn{2}{|c|}{ Univariate analysis } & \multicolumn{2}{|c|}{ Multivariate analysis } \\
\hline & HR $[95 \% I C]$ & $p$ value & HR $[95 \% I C]$ & $p$ value & HR [95\%IC] & $p$ value & HR $[95 \% I C]$ & $p$ value \\
\hline Orai3 (high vs. low) & $0.32[0.03-3.10]$ & 0.533 & & & $8.01[1.02-64.19]$ & 0.049 & & \\
\hline Gender (M vs. F) & $1.88[0.17-20.37]$ & 0.944 & & & $2.26[0.24-21.48]$ & 0.581 & & \\
\hline Necrosis (p vs. a) & $3.71[0.62-22.28]$ & 0.152 & & & $3.19[0.48-21.13]$ & 0.265 & & \\
\hline Age & 0.94 [0.79-1.09] & 0.929 & & & 1.08 [1.01-1.16] & 0.242 & & \\
\hline Tobacco use (s vs. nos) & $4.35[0.00-28.51]$ & 0.652 & & & $0.32[0.00-35.89]$ & 0.999 & & \\
\hline
\end{tabular}

Abbreviations: a: absence; 95\%IC: 95\% confidence interval; F: female; HR: Hazard Ratio; M: male; MFS: metastasis free survival; nos: non-smokers; OS: overall survival; p: presence; s: smokers.

was associated with poor prognosis (OS: 35.01 months $v s$. 51.11 months and MFS: 46.01 months vs. 62.04 months).

Furthermore, we showed that Orai3 expression is also associated with $\mathrm{ER} \alpha$ expression in lung adenocarcinoma such as demonstrated in breast adenocarcinoma [19]. Indeed, in breast cancer, Orai3 is overexpressed and its expression is under the control of estrogen receptor alpha $(\mathrm{ER} \alpha)$ that regulates $\mathrm{Ca}^{2+}$ entry, cell proliferation and survival of estrogen receptor-positive MCF-7 cell line [16, 17]. ER $\alpha$ is expressed in both tumor tissue and cultured non-small cell lung cancer (NSCLC) cell lines. $17-\beta$-estradiol increased cell proliferation in vitro and in vivo [27]. Moreover, suppressing expression of ER $\alpha$ elicits a significant reduction in NSCLC cell proliferation in vitro $[30,31]$. Furthermore, the association of $\mathrm{ER} \alpha$ with lung cancer is well established [28]. Clinically, the overexpression of ER $\alpha$ is associated with a poor prognosis in lung adenocarcinoma patients both for men and women [10, 29-31]. In animal model, enhanced E2/ER $\alpha$ facilitates the smoking carcinogen $\mathrm{N}$-nitrosamines-mediated lung carcinogenesis [32]. We demonstrated here an association between Orai3 expression and tobacco exposure. These findings suggested that Orai3 could be associated with tobacco smoke mediated ER $\alpha$ expression.

Postoperative chemotherapy is given to slow down or stop the growth of cancer cells. Even after a cancer has been removed with surgery, cancer cells can remain in the body, increasing the risk of relapse. Chemotherapy can eliminate these cancer cells and increase the chance of cure, but it is prescribed only to patients with a risk 
Table 4: Cox regression analysis for OS and MFS for adenocarcinoma patients for patients with II-IIIa stage (N=62)

\begin{tabular}{|c|c|c|c|c|c|c|c|c|}
\hline \multirow[t]{3}{*}{ Variable } & \multicolumn{4}{|c|}{ OS } & \multicolumn{4}{|c|}{ MPF } \\
\hline & \multicolumn{2}{|c|}{ Univariate analysis } & \multicolumn{2}{|c|}{ Multivariate Analysis } & \multicolumn{2}{|c|}{ Univariate analysis } & \multicolumn{2}{|c|}{ Multivariate analysis } \\
\hline & HR [95\%IC] & $p$ value & HR $[95 \% I C]$ & $p$ value & HR [95\%IC] & $p$ value & HR $[95 \% I C]$ & $p$ value \\
\hline Orai3 (high vs. low) & $4.61[1.61-13.17]$ & 0.004 & $7.16[1.69-30.34]$ & 0.008 & $1.82[0.78-4.25]$ & 0.168 & $1.37[0.57-3.28]$ & 0.479 \\
\hline Gender (M vs. F) & $1.16[0.42-3.19]$ & 0.334 & & & $0.66[0.27-1.61]$ & 0.307 & & \\
\hline Necrosis (p vs. a) & $1.98[0.91-4.34]$ & 0.086 & $1.72[0.78-3.78]$ & 0.176 & $3.20[1.42-7.20]$ & 0.005 & $2.94[1.29-6.71]$ & 0.011 \\
\hline Age & $1.02[0.97-1.06]$ & 0.379 & & & $0.96[0.92-1.01]$ & 0.221 & & \\
\hline Tobacco use (s vs. nos) & $1.29[0.26-6.55]$ & 1 & & & $0.33[0.09-1.17]$ & 0.824 & & \\
\hline
\end{tabular}

Abbreviations: a: absence; 95\%IC: 95\% confidence interval; F: female; HR: Hazard Ratio; M: male; MFS: metastasis free survival; nos: non-smokers; OS: overall survival; p: presence; s: smokers.

of recurrence high enough to counterbalance the side effects of chemotherapy. It is recommended for patients with stages II or III NSCLC and for some (but not all) patients with stage I disease. Reasonably the use of chemotherapy after radical surgery is justified by increasing overall survival from 60 to $64 \%$ [5-7]. As the benefit of chemotherapy is moderate, it is important to select appropriate patients with resectable tumours. Thus, patients are selected by using biomarkers that predict prognosis [33]. Adjuvant chemotherapy is prescribed only to the selected patients who are likely to benefit from the treatment $[34,35]$. The outcomes have showed that high Orai3 expression was associated with poor prognostic for MFS in Ib stage and with poor prognosis for OS in IIIII stage. This result supports high Orai3 expression as a good biomarker to select appropriate patients with Ib-II-III stage. Furthermore, the fact that higher Orai3 expression was found in III-IV stages vs. I-II stages $(\mathrm{N}=154$, $0.69 \pm 0.06 v s$. $\mathrm{N}=46,0.81 \pm 0.12$ ) support the use of Orai3 as therapeutic target.

Growing studies show the Orai3 pivotal role in mediating tumorigenesis. Indeed, two preclinical studies, in murine models, in breast and prostate cancers respectively have highlighted that inhibition of Orai3 impairs tumor growth and metastasis [36, 37] suggesting Orai 3 as a useful therapeutic target in oncology. Recently, a new compound targeting calcium release-activated calcium (CRAC) channels including Orai1 and Orai3 channels has been developed by Rhizen pharmaceuticals for the treatment of the nonsmall cell lung cancer (Patent US 2011/0112058 $A 1,[38])$. The compound named "B" decreases the expression of both Orai1 and Orai3 in NCl-H460 lung cancer cell line, and tumour growth in vivo [38, 39]. Although, this compound has affected both Orai1 and Orai3 expressions, these outcomes suggest that this drug could be a promising approach for cancer lung adenocarcinoma treatment.

In conclusion, Orai3 is associated with poor prognosis of lung adenocarcinoma and might be used as a novel prognostic marker for a chemotherapy treatment indication in early stage adenocarcinoma.

\section{MATERIALS AND METHODS}

\section{Ethics statement}

This study was conducted according to "Comité Consultatif de Protection des Personnes dans la Recherche Biomédicale de Picardie" in which 200 patients in resectable stage of lung adenocarcinoma were identified from the database of pathology department of Amiens University Hospital. Surviving patients provided their written consent for their clinical information to be included in the study.

\section{Specimens of study}

Patients with tumour and non-tumour samples between 2008 and 2013 have been included. Data entry was finalized on 2015. The tumors were classified according to the 2011 WHO classification [8]. 200 patients with lung adenocarcinoma were included at the time of the last follow-up. The median age of the patients at initial diagnosis was 60.5 years (range: $34-$ 84 years). The majority were male $(73 \%, \mathrm{~N}=143 / 200)$. $86.9 \%(\mathrm{~N}=173 / 200)$ of patients had tobacco exposure, defined by more than 100 cigarettes smoked in their lifetime.

\section{Immunohistochemical analysis}

The method for detecting and determining the expression of different proteins (Orai3 and estrogen receptor alpha) has been previously described [21]. Briefly, formalin-fixed, paraffin-embedded $4 \mu \mathrm{m}$ sections of the lung tissue were first deparaffinised in xylene and rehydrated in ethanol. The endogenous peroxidase activity was blocked (Ventana) before the antigen retrieval. The Cell Conditioning Solution 
CC1 (Ventana, BenchMark) was then used for antigen retrieval.

Immunohistochemical staining was carried out on BenchMark ULTRA (Ventana), using antibody directed against Orai3 (rabbit polyclonal, 1:200 dilution, Sigma, Saint Louis, USA) and antibody directed against Estrogen Receptor Alpha (ER $\alpha)$ (SP-1, Ventana, prediluted, Meylan, France). This was followed by the avidin-biotinperoxidase complex technique. Reactions were developed using a chromogenic reaction in DAB (diamino3,3benzidinetetrahy-drochloride) substrate solution (iVIEW DAB Detection Kit, Ventana). The tissues were counterstained with hematoxylin. The antibody is certified for immunohistochemistry by Sigma Inc. A negative control was performed using the same technique without the primary antibody.

\section{Quantification of staining expression by immunohistochemistry}

Independent observers quantitatively evaluated staining expression (x40 objective and $\mathrm{x} 10$ eyepiece) of each specimen. To evaluate relative immune-intensity in lung adenocarcinoma, immunoreactivity was also evaluated by $\mathrm{H}$ scoring system [40]. Briefly, staining carcinoma cells were further classified into the strongly or weakly positive cells, and $\mathrm{H}$ scores were subsequently generated by adding together $3 \mathrm{x} \%$ strongly stained cells, $2 \mathrm{x} \%$ moderate stained cells $1 \mathrm{x} \%$ weakly stained cells, and $0 \mathrm{x} \%$ negative cells. Thereafter, this staining score of Orai3 expression is referred to as the Orai3 expression level. Cases with ER $\alpha$ of $>1 \%$ were considered ER $\alpha$ positive for lung adenocarcinoma.

\section{Quantitative RT-PCR}

Every DNA sample was obtained from two 5 $\mu \mathrm{m}$-thick sections of non-tumour and tumour tissues. Extractions were performed manually according to manufacturers' protocols (ReliaPrep FFPE Total RNA Miniprep System, Promega Corp, France). Total RNA $(1 \mu \mathrm{g})$ was reverse transcribed into cDNA with random hexamers and MutliScribesTM Reverse Transcriptase (Applied Biosystems, Carlsbad, CA) as previously described [41]. Quantitative RT-PCR was performed on a Light Cycler system (Roche, Basel, Switzerland) using a mix containing SYBR green (Applied Biosystem, Carlsbad, CA). For the PCR reaction, sense and anti-sense PCR primers specific to Orai3 (for 5'-AAGTCAAAGCTTCCAGCCGC-3'; and rev 5'-GGTGGGTACTCGTGGTCACTCT-3') and $\beta$-actin (for 5'-CAGAGCAAGAGAGGCATCCCT-3'; and rev5'-ACGTACATGGCTGGGGTG-3') were used. The relative amount of Orai3 "target" was normalized to the endogenous control ( $\beta$-actin) and compared to the reference sample (non-tumour) using the Pfaffl method.

\section{Statistical analysis}

Data are presented as Mean \pm SEM. The MannWhitney U test was used to compare the Orai3 score between patients according to the presence or absence of variable, and the Wilcoxon signed-rank test was used to compare the distribution of expression among tissue types (non-tumour and tumour). Median survival was computed from the date of diagnosis to the date of the patient's last known vital status. The time to first metastasis was computed from the date of stage diagnosis to the date of first metastasis. A univariate and multivariate logistic regression model was built that included the following factors: tumour necrosis (presence $v s$. absence), tobacco exposition (smoker vs. no smoker), pleural visceral invasion (presence $v s$. absence), histologic classification of invasive adenocarcinoma (micropapillary or solid $v s$. lepidic, acinar or papillary predominant), expression of $\mathrm{ER} \alpha$ (expression $>1 \%$ vs. $<1 \%$ ), expression of TTF 1 (expression $>1 \%$ vs. $<1 \%$ ), EGFR (MT (Mutant Type) $v s$. WT (Wild Type)) and KRAS (MT vs. WT). This model was used to predict Orai3 expression. The Orai3 was considered first as a continuous variable, and secondary analyses were performed separately which categorized patients as having p-STAT3 expression $<1 \%$ vs. $>1 \%$ [17]. The log-rank test was used to assess groups with low Orai3 expression $(<1 \%) v s$. high $>1 \%)$. Cox proportional hazards regression was used to analyze the association between Orai3 expression and survival or the development of metastasis. We decided on this dichotomization based on its possible clinical relevance after analyzing absolutely none versus any Orai3 expression as well as comparing percentiles that represented the highest and lowest levels of expression. A p value of less than 0.05 was considered significant. All statistical analysis was performed using R.

\section{CONFLICTS OF INTEREST}

The authors declare that they have no interest of any kind affecting this study.

\section{FUNDING}

This study was supported by the Ministry of National Education (France), Amiens University Hospital, the Picardie biobank and "Ligue Nationale Contre le Cancer (SEPTENTRION) ».

\section{Authors' contribution}

B.N.: design, execution, interpretation of data, statistical analysis and manuscript preparation.

S.H.: execution and interpretation of data and manuscript preparation.

A.A. and O.A.H.: design and interpretation of data and manuscript preparation. 


\section{REFERENCES}

1. Torre LA, Bray F, Siegel RL, Ferlay J, Lortet-Tieulent J, Jemal A. Global cancer statistics, 2012. Cancer J Clin. 2015; 65: 87-108.

2. Roviello $\mathrm{G}$. The distinctive nature of adenocarcinoma of the lung. Onco Targets Ther. 2015; 8: 2399-2406.

3. Wright GM, Do H, Weiss J, Alam NZ, Rathi V, Walkiewwicz M, John T, Russell PA, Dobrovic A. Mapping of actionable mutations to histological subtype domains in lung adenocarcinoma: implications for precision medicine.Oncotarget. 2014; 5: 2107-2115. doi: 10.18632/ oncotarget. 1840 .

4. Sica G, Yoshizawa A, Sima CS, Azzoli CG, Downey RJ, Rusch VW, Travis WD, Moreira AL. A grading system of lung adenocarcinomas based on histologic pattern is predictive of disease recurrence in stage I tumours. Am J Surg Pathol. 2010; 34: 1155-1162.

5. Burdett S, Pignon JP, Tierney J, Tribodet H, Stewart L, Le Pechoux C, Aupérin A, Le Chevalier T, Stephens RJ, Arriagada R, Higgins JP, Johnson DH, Van Meerbeeck J, et al. Adjuvant chemotherapy for resected early-stage non-small cell lung cancer. Cochrane Database Syst Rev. 2015; 3: CD011430.

6. Burdett S, Stewart LA, Rydzewska L. A systematic review and meta-analysis of the literature: chemotherapy and surgery versus surgery alone in non-small cell lung cancer. J Thorac Oncol. 2006; 1: 611-621.

7. Berghmans T, Meert AP, Martin B, Ninane V, Sculier JP. Prognostic role of epidermal growth factor receptor in stage III nonsmall cell lung cancer. Eur Respir J. 2005; 25: 329-335.

8. Travis WD, Brambilla E, Noguchi M, Nicholson AG, Geisinger KR, Yatabe Y, Beer DG, Powell CA, Riely GJ, Van Schil PE, Garg K, Austin JH, Asamura H, et al. International association for the study of lung cancer/ american thoracic society/european respiratory society international multidisciplinary classification of lung adenocarcinoma. J Thorac Oncol. 2011; 6: 244-285.

9. Kawai H. Estrogen receptors as the novel therapeutic biomarker in non-small cell lung cancer. World J Clin Oncol. 2014; 5: 1020-1027.

10. Shimizu K, Hirami Y, Saisho S, Yukawa T, Maeda A, Yasuda K, Nakata M. Membrane-bound estrogen receptor- $\alpha$ expression and epidermal growth factor receptor mutation are associated with a poor prognosis in lung adenocarcinoma patients. World J Surg Oncol. 2012; 10: 141.

11. Kadota K, Eguchi T, Villena-Vargas J, Woo KM, Sima CS, Jones DR, Travis WD, Adusumilli PS. Nuclear estrogen receptor- $\alpha$ expression is an independent predictor of recurrence in male patients with pT1aN0 lung adenocarcinomas, and correlates with regulatory T-cell infiltration. Oncotarget. 2015; 6: 27505-27518. doi: 10.18632/oncotarget.4752.

12. Capiod T. The need for calcium channels in cell proliferation. Recent Pat Anticancer Drug Discov. 2013; 8: 4-17.
13. Pande G, Kumar NA, Manogaran PS. Flow cytometric study of changes in the intracellular free calcium during the cell cycle. Cytometry. 1996; 24: 55-63.

14. Kondratskyi A, Kondratska K, Skryma R, Prevarskaya $\mathrm{N}$. Ion channels in the regulation of apoptosis. Biochim Biophys Acta. 2015; 1848: 2532-2546.

15. Shuttleworth TJ. Orai3--the 'exceptional' Orai? J Physiol. 2012; 590: 241-257.

16. Motiani RK, Zhang $\mathrm{X}$, Harmon KE, Keller RS, Matrougui K, Bennett JA, Trebak M. Orai3 is an estrogen receptor alpha-regulated $\mathrm{Ca} 2+$ channel that promotes tumourigenesis. FASEB J. 2013; 27: 63-75.

17. Faouzi M, Kischel P, Hague F, Ahidouch A, Benzerdjeb N, Sevestre H, Penner R, Ouadid-Ahidouch H. ORAI3 silencing alters cell proliferation and cell cycle progression via c-myc pathway in breast cancer cells. Biochim Biophys Acta. 2013; 1833: 752-760.

18. Dubois C, Vandenabeele F, Lehen'kyi V, Gkika D, Guarmit B, Lepage G, Slomianny C, Borowiec AS, Bidaux G, Benahmed M, Shuba Y, Prevarskaya N. Remodeling of channel-forming ORAI proteins determines an oncogenic switch in prostate cancer. Cancer Cell. 2014; 26: 19-32.

19. Motiani RK, Abdullaev IF, Trebak M. A novel native storeoperated calcium channel encoded by Orai3: selective requirement of Orai3 versus Orai1 in estrogen receptorpositive versus estrogen receptor-negative breast cancer cells. J Biol Chem. 2010; 285: 19173-19183.

20. Faouzi M, Hague F, Potier M, Ahidouch A, Sevestre H, Ouadid-Ahidouch H. Down-regulation of Orai3 arrests cellcycle progression and induces apoptosis in breast cancer cells but not in normal breast epithelial cells. J Cell Physiol. 2011; 226: 542-551.

21. Ay AS, Benzerdjeb N, Sevestre H, Ahidouch A, OuadidAhidouch H. Orai3 constitutes a native store-operated calcium entry that regulates non-small cell lung adenocarcinoma cell proliferation. PLoS One. 2013; 8: e72889.

22. Detre S, SaclaniJotti G, Dowsett M. A "quickscore" method for immunohistochemical semiquantitation: validation for oestrogen receptor in breast carcinomas. J Clin Pathol. 1995; 48: 876-878.

23. Chen Z, Fillmore CM, Hammerman PS, Kim CF, Wong KK. Non-small-cell lung cancers: a heterogeneous set of diseases. Nat Rev Cancer. 2014; 14: 535-546.

24. Jakobsen JN, Santoni-Rugiu E, Ravn J, Sorensen JB. Intratumour variation of biomarker expression by immunohistochemistry in resectable non-small cell lung cancer. Eur J Cancer. 2013; 49: 2494-2503.

25. Ko JH, Gu W, Lim I, Bang H, Ko EA, Zhou T. Ion channel gene expression in lung adenocarcinoma: potential role in prognosis and diagnosis. PLoS One. 2014; 9: e86569.

26. Grills C, Jithesh PV, Blayney J, Zhang SD, Fennell DA. Gene expression meta-analysis identifies VDAC1 as a predictor of poor outcome in early stage non-small cell lung cancer. PLoS One. 2011; 6: e14635. 
27. Stabile LP, Siegfried JM. Estrogen receptor pathways in lung cancer. Curr Oncol Rep. 2004; 6: 259-267.

28. Márquez-Garbán DC, Chen HW, Fishbein MC, Goodglick L, Pietras RJ. Estrogen receptor signaling pathways in human non-small cell lung cancer. Steroids. 2007; 72: 135-143.

29. Kawai H, Ishii A, Washiya K, Konno T, Kon H, Yamaya C, Ono I, Minamiya Y, Ogawa J. Estrogen receptor alpha and beta are prognostic factors in non-small cell lung cancer. Clin Cancer Res. 2005; 11: 5084-5089.

30. Rades D, Setter C, Dahl O, Schild SE, Noack F. The prognostic impact of tumor cell expression of estrogen receptor-alpha, progesterone receptor, and androgen receptor in patients irradiated for non small cell lung cancer. Cancer. 2012; 118: 157-163.

31. Olivo-Marston SE, Mechanic LE, Mollerup S, Bowman ED, Remaley AT, Forman MR, Skaug V, Zheng YL, Haugen A, Harris CC. Serum estrogen and tumor-positive estrogen receptor-alpha are strong prognostic classifiers of nonsmall-cell lung cancer survival in both men and women. Carcinogenesis. 2010; 31: 1778-1786.

32. Li MY, Liu Y, Liu LZ, Kong AW, Zhao Z, Wu B, Long X, Wu J, Ng CS, Wan IY, Du J, Mok TS, Underwood MJ, et al. Estrogen receptor alpha promotes smoking-carcinogeninduced lung carcinogenesis via cytochrome P450 1B1. J Mol Med. 2015; 93: 1221-1233.

33. Tanaka F, Yoneda K. Adjuvant therapy following surgery in non-small cell lung cancer (NSCLC). Surg Today. 2016; 46:25-37.

34. Massuti B, Sanchez JM, Hernando-Trancho F, Karachaliou N, Rosell R. Are we ready to use biomarkers for staging, prognosis and treatment selection in early-stage non-smallcell lung cancer? Transl Lung Cancer Res. 2013; 2: 208-221.
35. Wallerek S, Sorensen JB. Biomarkers for efficacy of adjuvant chemotherapy following complete resection in NSCLC stages I-IIIA. Eur Respir Rev. 2015; 24: 340-355.

36. Motiani RK, Zhang X, Harmon KE, Keller RS, Matrougui $\mathrm{K}$, Bennett JA, Trebak M. Orai3 is an estrogen receptor $\alpha$-regulated $\mathrm{Ca}^{2+}$ channel that promotes tumorigenesis. FASEB J. 2013; 27:63-75.

37. Dubois C, Vanden Abeele F, Lehen'kyi V, Gkika D, Guarmit B, Lepage G, Slomianny C, Borowiec AS, Bidaux G, Benahmed M, Shuba Y, Prevarskaya N. Remodeling of channel-forming ORAI proteins determines an oncogenic switch in prostate cancer. Cancer Cell. 2014; 26:19-32.

38. Muthuppalaniappan M, Viswanadha S, Varanasi KV, Merikapudi GS, Vakkalanka SK. Novel modulators of calcium release-activated calcium channel and methods for treatment of non-small cell lung cancer. US20110112058 A1 Patent, 2011.

39. Munaron L, Genova T, Avanzato D, Antoniotti S, FiorioPla A. Targeting calcium channels to block tumour vascularization. Recent Pat Anticancer Drug Discov. 2013; 8: 27-37.

40. Mazières J, Brugger W, Cappuzzo F, Middel P, Frosch A, Bara I, Klingelschmitt G, Klughammer B. Evaluation of EGFR protein expression by immunohistochemistry using H-score and the magnification rule: re-analysis of the SATURN study. Lung Cancer. 2013; 82: 231-237.

41. Rybarczyk P, Gautier M, Hague F, Dhennin-Duthille I, Chatelain D, Kerr-Conte J, Pattou F, Regimbeau JM, Sevestre H, Ouadid-Ahidouch H. Transient receptor potential melastatin-related 7 channel is overexpressed in human pancreatic ductal adenocarcinomas and regulates human pancreatic cancer cell migration. Int J Cancer. 2012; 131: E851-E861. 